21st Particles and Nuclei International Conference (PANIC 2017)

International Journal of Modern Physics: Conference Series

Vol. 46 (2018) 1860059 (5 pages)

(C) The Author(s)

DOI: $10.1142 / \mathrm{S} 2010194518600595$

\title{
On a Possibility of the Gravitational Wave Detection at the High Energy Colliders
}

\author{
Murli Manohar Verma \\ Department of Physics, University of Lucknow, \\ Lucknow, 226007, India \\ sunilmmv@yahoo.com
}

Published 3 May 2018

\begin{abstract}
A strong follow up of a previous proposal (ICHEP, Valencia 2014) is made leading to the first experiment to observe the gravitational waves at the collision sites at the colliders such as the Large Hadron Collider at CERN. The amplitudes have been calculated with regard to the sensitivity of the detector. Compared with the standard model physics, it is shown to have a measurable impact on the particle motions and corresponds to 'missing' energy in form of the gravitational wave loss. This is unlike the cosmological detectors like BICEP2 etc. where the indirect B mode polarization on CMBR were masked by dust. In contrast, this experiment would be the first experiment where the energy-momentum tensor of the source can be controlled.
\end{abstract}

Keywords: Gravitational waves; collider; Higgs field.

\section{Why Look For the Gravitational Waves at Colliders?}

The core idea behind the recent detection of gravitational waves in cosmology, such as the most recent GW170817, has been to observe the spacetime ripples propagating away from the objects having oscillating quadrupole moment in the cataclysmic events within a pair of the black hole or neutron star collisions. The cosmological observations taken from the ground based detectors as aLIGO and VIRGO, as well as gamma, X-ray and radio observations have opened an area of active research in multi-wavelength astronomy for successive follow up leading to the construction of viable models of mergers and structure of such objects. In addition to it, we have the gravitational waves of the inflationary origin in the early universe that are not directly detectable unlike those from the astrophysical sources, but leave their signatures in form of the B mode polarization of the Cosmic Microwave Background

This is an Open Access article published by World Scientific Publishing Company. It is distributed under the terms of the Creative Commons Attribution 4.0 (CC-BY) License. Further distribution of this work is permitted, provided the original work is properly cited. 
Radiation. However, as in the recent observations, we are forced to wait for the signals to arrive while sitting in the waiting lounge with our interferometers and be able to observe only as and when they arrive at our detectors. Considering the factors of intrinsically weak strength of the gravitational waves, and the enormous cosmological distances they have to travel through space before they reach the ground-based detectors brings in a great deal of difficulty in their observation. Also, as we cannot control the source conditions or en-route parameters, we have little to experiment with. This gives a reason to think in terms of a future experiment that can be done with the colliders where at the highest energy collision sites the events, though extremely small on energy scales compared with the cosmic mergers, are located at the distances extremely close compared with their cosmic counterparts. These two counter effects related to the magnitude of energy scales and the distances would offset each other in colliders, along with the added benefit of the experimenter's freedom to control the parameters of the experiment, like event energy scale, distance or angular dependence of the propagation of the gravitational waves, and also the accuracy and precision of measurements can be quite high even under natural constraints.

Previously, it was shown that one can find a single Higgs-like field to relate the early inflation and the present acceleration of the universe. Using this field, we obtained a single source of the gravitational waves from the early inflation, as well as from the recent accelerated phase of the universe in the Higgs-like field. The Higgs boson discovery at the Large Hadron Collider is another motivation towards the existence of this possible relationship and also to look for its effects in form of gravitational waves. We can express the action of the Higgs-like scalar field as (tachyonic action) ${ }^{1}$

$$
\mathcal{A}=\int d^{4} x \sqrt{-g}\left(\frac{R}{16 \pi G}-V(\phi) \sqrt{1-\partial^{i} \phi \partial_{i} \phi}\right)
$$

The Lagrangian can be splitted with pressure and energy density as

$$
\begin{gathered}
P=-\frac{V(\phi)}{\sqrt{1-\dot{\phi}^{2}}}+\frac{\dot{\phi}^{2} V(\phi)}{\sqrt{1-\dot{\phi}^{2}}}+0 \\
\rho=\frac{V(\phi)}{\sqrt{1-\dot{\phi}^{2}}}+\frac{3 \dot{\phi}^{2} V(\phi)}{\sqrt{1-\dot{\phi}^{2}}}-\frac{3 \dot{\phi}^{2} V(\phi)}{\sqrt{1-\dot{\phi}^{2}}} .
\end{gathered}
$$

It is seen from (2) and (3) that the inclusion of radiation in tachyonic scalar field gives rise to appearance of new exotic component (with negative energy density and zero pressure). Thus, the tachyonic scalar field breaks into three components. In our previous work, ${ }^{2}$ a time dependent change was studied in the equation of state (EoS) of the cosmological constant with

$$
\bar{w}_{\lambda}=-1+\varepsilon(t) .
$$


Thus, with the perturbed EoS, the cosmological constant (with varying energy density but fixed EoS) becomes a shifted cosmological parameter with varying EoS. With fixed energy density of field components, the expressions for the energy density and pressure of each component are given as below. For the shifted cosmological constant one has

$$
\begin{gathered}
\bar{\rho}_{\lambda}=\frac{V(\phi)}{\sqrt{1-\dot{\phi}^{2}}} \\
\bar{p}_{\lambda}=\frac{-V(\phi)}{\sqrt{1-\dot{\phi}^{2}}}+\frac{\varepsilon V(\phi)}{\sqrt{1-\dot{\phi}^{2}}}
\end{gathered}
$$

and

$$
\bar{w}_{\lambda}=-1+\varepsilon(t)
$$

In case of the shifted radiation it becomes

$$
\begin{gathered}
\bar{\rho}_{r}=\frac{3 \dot{\phi}^{2} V(\phi)}{\sqrt{1-\dot{\phi}^{2}}} \\
\bar{p}_{r}=\frac{(1+3 \varepsilon) \dot{\phi}^{2} V(\phi)}{\sqrt{1-\dot{\phi}^{2}}}
\end{gathered}
$$

with

$$
\bar{w}_{r}=\frac{1}{3}+\varepsilon .
$$

The perturbation changes the pressureless exotic matter into fluid with negative non-zero pressure

$$
\begin{gathered}
\bar{\rho}_{m}=\frac{-3 \dot{\phi}^{2} V(\phi)}{\sqrt{1-\dot{\phi}^{2}}} \\
\bar{p}_{m}=p_{\phi}-\bar{p}_{\lambda}-\bar{p}_{r}=\frac{-\varepsilon\left(1+3 \dot{\phi}^{2}\right) V(\phi)}{\sqrt{1-\dot{\phi}^{2}}}
\end{gathered}
$$

with

$$
\bar{w}_{m}=\frac{\varepsilon\left(1+3 \dot{\phi}^{2}\right)}{3 \dot{\phi}^{2}} .
$$




\section{The gravitational waves at the Large Hadron Collider}

We had found earlier that around $l=100 B$-mode power spectrum in the BICEP2 results must have the inputs from the late-time acceleration to tensor-to-scalar ratio $r \simeq 0.2$. Gravitational waves from the present acceleration must induce further quadrupole anisotropies into the $B$-modes, in addition to those caused by early inflation, and other galactic dust effects that convert $E$ - to $B$ - modes.

Here we also propose setting up a GW detector at the LHC collision site. The following points are in order-

(i) The GW detector around the LHC beam may be in form of a spherical shell with collisions at the centre. As the GW of frequency between $10^{-7}$ to $10^{7} \mathrm{~Hz}$ propagates the space around it will oscillate, and so with it practically, any material particles would oscillate with such time order, and as these particles (which are at present produced in the ATLAS/CMS) move away they would show a wobbling motion outwards.

In fact, it must bend the paths of laser light beams (or the existing radiation tracks) around in the similar way on the same time scale!

The effects could thus be cross-checked in the GW detector directly, as well as the distortion/wobbling effects on particles and radiation geodesics emanating which are not explained by the particle physics per se in the existing experiments.

(ii) The distances over which a wavelength must come in full may be around a few millimetres. The detection will be made continuously over a few metres. The amplitude will decay with distance, but luckily, it would give much better dividends than the cosmological detectors like BICEP2 that must wait and watch out for the extremely feeble signals, that travelled over billions of years and whose indirect imprints of polarization are indelibly masked by several spurious effects. Here, we will be doing an experiment in true sense by controlling the stress energy tensor (and so the amplitudes of perturbations) of the sources.

(iii) Any effects of cosmic dust on polarization where BICEP2 slipped and must obscure the GW signatures in cosmological detectors are absent in our case of the LHC GW detector. Bringing cosmology into particle accelerator would be a novel idea. The LHC is just right place to do that.

(iv) The calculation of the strain and spectral amplitude (power spectral density) of the gravitational waves at the LHC as proposed has been done. The lowest order Post-Newtonian approximation (as in general relativity the linearization must be used) calculations indicate that the measurable amplitude may be above $10^{-20}$ at the collision sites. It is hopefully not too hard to measure, by further dependence on the actual beam energy density in CM frame.

(v) The frequencies from transient (bursting) sources may spread out in the range $(2-4) 10^{7} \mathrm{~Hz}$. So, this will be high frequency range of GW much beyond the cosmological detectors (the ground based like LIGO/GEO/VIRGO have it around $10^{2} \mathrm{~Hz}$. while the CMBR polarizations correspond to a very low 
frequency of $10^{-16} \mathrm{~Hz}$ ). The amplitude, as estimated from the quadrupole formula as the second moment of mass distribution gives $h=2 / r<$ energy $>$.

(vi) To detect GW, the interferometric setup at least of the size of the wavelength would be the most optimum attainable by observing the shifts in the interference fringes (to remove any contribution of stationary part of gravitational field).

\section{Conclusions}

In nut-shell, Higgs-like cosmological field splits into parts and the components interact mutually. Lambda decays into dark matter. Recent acceleration produces its own gravitational waves (GW) superposed on the primordial GW generated by the inflation. This results in enhanced tensor-to-scalar ratio of amplitudes observed in overall BICEP2 data. Under the planned energy scales of 16-18 TeV in Run 2 of the Large Hadron Collider, the highest to date, it could be a potential site for production and observation of GW due to associated tensor perturbations in spacetime. This would be of course an alternative search to cosmological detectors like BICEP2, which have no option but to wait for the indirect signatures of the extremely feeble GW imprints on the Cosmic Microwave Background. Even these weak signatures are masked by dust polarization and so causes model-based confusion in misinterpretations. Here at LHC, one can manipulate the source of energy tensor and set up a GW detector close to the collision site. The GW frequency range may be $10^{-10}-10^{10} \mathrm{~Hz}$ and amplitude may be above $10^{-20}$ at the collision sites.

\section{Acknowledgments}

The author thanks Albert de Roeck at CERN for the initial discussions and the Inter-University Centre for Astronomy and Astrophysics, Pune for the support and facilities under the associateship programme.

\section{References}

1. M. M. Verma and S. D. Pathak, Int. J. Mod. Phys. D 23, 9, 1450075(2014).

2. M. M. Verma and S. D. Pathak, Astrophys. Space Sci. 344, 505(2013).

3. P. A. R. Ade et al., Phys. Rev. Lett. B 112, 241101(2014).

4. P. A. R Ade et al., Planck Collaboration (2013), arxiv:1303.5082.

5. M. M. Verma, Astrophys. Space Sci. 330, 101(2010). 